\title{
Potencial produtivo de novas seleções de nespereiras
}

\author{
Production of news selections of loquat tree
}

\section{José Emílio Bettiol Neto' ${ }^{\mathrm{I}}$ Fernando Antônio Campo Dall’Orto ${ }^{\mathrm{I}}$ Wilson Barbosa ${ }^{\mathrm{II}}$ Edvan Alves Chagas ${ }^{\mathrm{III}}$ Rafael Pio $^{\mathrm{IV} *}$ Fernanda Carvalho Costa ${ }^{\mathrm{IV}}$}

\section{RESUMO}

Foi avaliada a capacidade produtiva das seleções de nêspera (Eriobotrya japonica) IAC 263-49, IAC 363-27, IAC 264-54, IAC 464-9, IAC 165-9, IAC 265-66, IAC 966-23, IAC 167-1, IAC 677-13, IAC 967-5, IAC 1367-43, IAC 136746, IAC 1467-12, IAC 1467-25, IAC 1467-170, IAC 1467177, IAC 1467-296, IAC 1467-301, IAC NE-3, IAC KM-6 e 'Mizuho', em Jundiaí, São Paulo, Brasil. As mudas, formadas com porta-enxertos de 'Mizumo', foram plantadas a campo, em blocos ao acaso. Do terceiro ao sétimo ano do plantio, foram quantificados o número e a massa fresca dos frutos, a produção total por planta e a produtividade estimada. Além disso, foi feito o cálculo da média do quadriênio. As seleções IAC 165-9, IAC 265-66 e IAC 677-13 apresentaram as maiores produções e são promissoras para a diversificação do cultivo de nêspera.

Palavras-chave: Eriobotrya japonica Lindl., nêspera, qualidade de fruto.

\section{ABSTRACT}

The yield of the loquat tree selections (Eriobotrya japonica) IAC 263-49, IAC 363-27, IAC 264-54, IAC 464-9, IAC 165-9, IAC 265-66, IAC 966-23, IAC 167-1, IAC 677-13, IAC 967-5, IAC 1367-43, IAC 1367-46, IAC 1467-12, IAC 1467-25, IAC 1467-170, IAC 1467-177, IAC 1467-296, IAC 1467-301, IAC NE-3, IAC KM-6 and 'Mizuho' were evaluated, in Jundiaí city, SP, Brazil. The seedlings growing on cultivar 'Mizumo' rootstocks were planted at field in a randomized blocks design. From the third to seventh year after planting the fruits number and fresh weight, the total yield per plant and the productivity were evaluated, besides the calculation of the average of the quadriennium. The IAC 165-9, IAC 265-66 and IAC 677-13 selections showed the highest productions and they are promising for the diversification of the loquat tree cultivation.

Key words: Eriobotrya japonica Lindl., loquat, fruit quality.

\section{INTRODUÇÃO}

O cultivo de nêsperas (Eriobotrya japonica) vem se intensificando no mundo, em especial nos países europeus localizados na região do mediterrâneo e no Brasil, pela excelente qualidade organoléptica de seus frutos, pela baixa necessidade de aplicações sistemáticas de defensivos agrícolas, frente aos mínimos problemas fitossanitários, e pela excelente fonte de renda, em razão do crescente consumo nos últimos anos (PIO et al., 2008).

O aumento do consumo de nêsperas está relacionado principalmente ao sabor peculiar dos seus frutos, bem como as suas características nutracêuticas. As nêsperas são ricas em ácido galacturônico, málico e fumárico (FEMENIA et al., 1998), flavonoides, carotenoides e outros e compostos antioxidantes, localizados nas casca e na polpa dos frutos (KOBA et al., 2007; FARIA et al., 2009; FERRERES et al., 2009). Além disso, possui elevada concentração de pectina

ICentro Avançado de Pesquisa Tecnológica do Agronegócio de Frutas, Instituto Agronômico (IAC), 13214-820, Jundiaí, SP, Brasil.

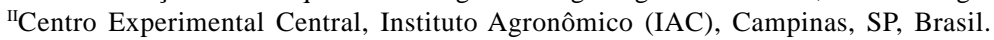

IIIEmpresa Brasileira de Pesquisa Agropecuária (EMBRAPA CPAFRR), Boa Vista, RR, Brasil.

IvUniversidade Federal de Lavras (UFLA), CP 3037, 37200-000, Lavras, MG, Brasil. E-mail: rafaelpio@dag.ufla.br. *Autor para correspondência. 
(MELO \& LIMA, 2003), o que vem a favorecer o processamento industrial na fabricação de doces, como geléias e compotas, atividade ainda não explorada comercialmente (PIO et al., 2008).

No Brasil, é uma excelente opção de cultivo para a diversificação de propriedades rurais, principalmente no que tange à época de maturação de seus frutos, que se concentra entre os meses de julho e setembro, quando há escassez de outras frutas no mercado (GRASSI et al., 2010).

O Estado de São Paulo, maior produtor nacional de nêsperas, possui cerca de 320 mil plantas em cultivo, com uma produção anual de 18,5mil t, e 85\% delas concentram-se na região do Alto Tietê (Mogi das Cruzes, Guararema, Biritiba-Mirim, Salesópolis e Santa Isabel), com 250ha cultivados (PEROSA et al., 2006). Além de Mogi das Cruzes, a nespereira é encontrada em outros 26 municípios do Estado de São Paulo, se constituindo na oitava frutífera de clima temperado-subtropical mais cultivada em São Paulo (BARBOSA et al., 2003).

A morfologia dos frutos das nêsperas varia consideravelmente, dependendo de sua origem e cultivar, podendo apresentar forma desde esférica até piriforme e coloração da casca de amarelo-pálida a alaranjado-forte (OJIMA et al., 1999). Em experimento realizado em Jundiaí, São Paulo (SP), se verificou que a massa unitária de cinco cultivares variaram de 32,61 a 25,83g e as nespereiras 'Mizuho', 'Mizumo', 'Mizauto' e 'Néctar de Cristal' apresentaram os maiores rendimentos de frutos, entre 14,84 e 15,81 kg planta ${ }^{-1}$, média dos quatro primeiros anos de produção (PIO et al., 2007a). Porém, as principais cultivares utilizadas pelos produtores brasileiros são 'Mizuho', 'Precoce de Itaquera' ('Fukuhara’) e ‘Precoce de Campinas’ (IAC 165-31) (BARBOSA et al., 2003).

Apesar de a nespereira possuir algumas vantagens de cultivo em relação a outras frutíferas, a lucratividade ainda é baixa, em razão de problemas relacionados ao desempenho produtivo das cultivares disponíveis aos produtores e ainda problemas de logística (PEROSA et al., 2006). A disponibilização de novas seleções avançadas é primordial para o cultivo em São Paulo, principalmente no que tange aos incrementos produtivos, para assim se elevar a renda em unidades produtoras dessa frutífera.

O presente trabalho teve como objetivo quantificar o desempenho produtivo de novas seleções de nespereira, nas condições do Município de Jundiaí, SP.

\section{MATERIAL E MÉTODOS}

Sementes da nespereira 'Mizumo' foram extraídas de frutos maduros, coletados em meados de maio de 2001 para a produção dos porta-enxertos. Após a extração, foram lavadas em água corrente, secas à sombra por 48 horas, semeadas sob leito de areia em viveiro coberto com sombrite de náilon (50\% de luminosidade) e irrigadas diariamente. Passados 90 dias da semeadura, as plântulas foram transplantadas para sacos plásticos pretos (dimensões de $18 \times 30 \mathrm{~cm}$, capacidade de três litros de substrato) e permaneceram nas mesmas condições de viveiro.

Em maio de 2002, quando os porta-enxertos estavam aptos para a realização da enxertia $(70 \mathrm{~cm}$ de altura e diâmetro de $12 \mathrm{~mm}$, em média), estas foram enxertadas por garfagem do tipo inglês complicado, a $15 \mathrm{~cm}$ de altura, utilizando garfos com três gemas, e coletadas das seguintes seleções avançadas de nespereiras em lote de segunda seleção: IAC 263-49, IAC 363-27, IAC 264-54, IAC 464-9, IAC 165-9, IAC 265-66, IAC 966-23, IAC 167-1, IAC 677-13, IAC 967-5, IAC 1367-43, IAC 1367-46, IAC 1467-12, IAC 1467-25, IAC 1467-170, IAC 1467-177, IAC 1467-296, IAC 1467301, IAC NE-3, IAC KM-6 e 'Mizuho’ (testemunha com elevado padrão produtivo). Com o intuito de evitar a dessecação do material propagativo, os garfos enxertados foram protegidos com sacos plásticos transparentes e amarrados com barbante durante 30 dias (OJIMA et al., 1999).

Após cinco meses da enxertia, as mudas foram levadas a campo, no espaçamento 7 x $4 \mathrm{~m}$. O experimento foi implantado em área do Centro Avançado de Pesquisa Tecnologia do Agronegócio das Frutas, do Instituto Agronômico (IAC), em Jundiaí, SP (238'S; 46 55’W; altitude de 700m). O clima da região, segundo classificação de Köeppen, é classificado como mesotérmico de inverno seco (Cwa), comumente chamado de tropical de altitude, com temperatura anual média de $21,4^{\circ} \mathrm{C}$ (média mínima de $15,3^{\circ} \mathrm{C}$ e média máxima de $27,4^{\circ} \mathrm{C}$ ) e precipitação média anual de $1.400 \mathrm{~mm}$. O solo, no local do experimento, é raso, pouco desenvolvido e bem drenado, identificado como unidade Currupira-modal (Cur), pertencente ao grande grupo Litosol, fase substrato filito-xisto (Embrapa, 2006). As plantas foram conduzidas segundo as recomendações de OJIMA et al. (1999), sendo adubadas anualmente, de acordo com análises de solo, e pulverizadas com fungicidas e inseticidas, quando necessário.

Ciência Rural, v.40, n.6, jun, 2010. 
O delineamento utilizado foi em blocos ao acaso, com 21 tratamentos, quatro blocos e cinco plantas por unidade experimental, sendo apenas consideradas para as análises as três plantas centrais. Entre os anos agrícolas de 2005 e 2008, foram quantificados a produção média por planta, o número médio de frutos, a massa fresca média dos frutos e a produtividade estimada. Ao final da última avaliação, calcularam-se as médias do quadriênio. As colheitas foram realizadas entre junho e meados de outubro, considerando os três picos anuais de maturação dos frutos.

Os dados foram submetidos à análise de variância, e as médias foram comparadas pelo teste Scott-Knott $(\mathrm{P} \leq 0,05)$. As análises foram realizadas pelo programa computacional Sistema para Análise de Variância-SISVAR(FERREIRA, 2000).

\section{RESULTADOS E DISCUSSÃO}

As seleções IAC 464-9, IAC 165-9 e IAC 677-13 se destacaram já no primeiro ano de avaliação quanto ao número médio de frutos, e apenas as duas primeiras citadas mantiveram o mesmo destaque no segundo ano de avaliação (Tabela 1). Na avaliação final, as seleções IAC 165-9 e IAC 677-13 produziram mais de mil frutos por planta, o dobro obtido pela nespereira 'Mizuho' e ainda três vezes mais que a 'Centenária' (389 frutos), de acordo com os resultados obtidos por PIO et al. (2007a). Essas duas seleções foram as que registraram a maior média do quadriênio.

Em relação à massa fresca média dos frutos, os resultados variaram muito de um ano para outro, com exceção da seleção IAC 265-66, que se destacou na primeira, na segunda e na quarta avaliação, apresentando assim a maior média do quadriênio (Tabela 2). De forma geral, as seleções e mesmo a nespereira 'Mizuho' não apresentaram frutos de elevado calibre, no máximo, 38,11 g, com a seleção IAC 265-66 no segundo ano de avaliação. No entanto, a literatura cita o emprego de técnicas auxiliares que venham a promover um melhor crescimento das frutas, como a aplicação da auxina 2,4-D, a 25mg L-1 (AGUSTÍ et al., 2003), o que poderá ser pesquisado para as cultivares de nêspera selecionada no Brasil.

As seleções IAC 464-9, IAC 165-9, IAC 26566, IAC 677-13 e IAC 1467-170 apresentaram maior produção média no primeiro ano de avaliação, não

Tabela 1 - Número médio de frutos por planta de novas seleções de nespereira. Centro APTA Frutas/IAC, Jundiaí, SP, 2009.

\begin{tabular}{|c|c|c|c|c|c|}
\hline \multirow{3}{*}{$\begin{array}{l}\text { Seleções } \\
\text { IAC 263-49 }\end{array}$} & \multirow[b]{2}{*}{2005} & \multirow[b]{2}{*}{2006} & \multirow[b]{2}{*}{2007} & \multirow[b]{2}{*}{2008} & \multirow[b]{2}{*}{ Média do quadriênio } \\
\hline & & & & & \\
\hline & 120 & 208 & 555 c & $533 \mathrm{c}$ & $354 \mathrm{~d}$ \\
\hline IAC 363-27 & 78 d & 354 & $682 \mathrm{c}$ & $651 \mathrm{c}$ & 441 c \\
\hline IAC 264-54 & $215 \mathrm{~b}$ & $467 \mathrm{~d}$ & 965 a & $633 \mathrm{c}$ & $570 \mathrm{~b}$ \\
\hline IAC 464-9 & 383 a & 772 a & 846 b & 869 b & $718 \mathrm{~b}$ \\
\hline IAC 165-9 & 426 a & 858 a & 1000 a & 1125 a & 852 a \\
\hline IAC 265-66 & $225 \mathrm{~b}$ & 523 с & 812 b & 855 b & $611 \mathrm{~b}$ \\
\hline IAC 966-23 & $138 \mathrm{c}$ & 509 & 746 b & $655 \mathrm{c}$ & $512 \mathrm{~b}$ \\
\hline IAC 167-1 & 138 & 363 & $459 \mathrm{~d}$ & 571 c & $383 \mathrm{c}$ \\
\hline IAC 677-13 & 347 a & $689 \mathrm{~b}$ & 1041 a & 1117 a & 798 a \\
\hline IAC 967-5 & $169 \mathrm{c}$ & 354 e & 555 c & 552 c & $407 \mathrm{c}$ \\
\hline IAC 1367-43 & 208 b & 417 & $553 \mathrm{c}$ & 581 c & 440 \\
\hline IAC 1367-46 & $217 b$ & 375 & 585 c & 595 & 443 \\
\hline IAC 1467-12 & $172 \mathrm{c}$ & 463 & 816 b & $701 \mathrm{c}$ & $534 \mathrm{~b}$ \\
\hline IAC 1467-25 & 222 b & 533 c & 744 b & $571 \quad c$ & 517 b \\
\hline IAC $1467-170$ & $263 \mathrm{~b}$ & $688 \mathrm{~b}$ & 972 а & $570 \quad \mathrm{c}$ & $623 \mathrm{~b}$ \\
\hline IAC 1467-177 & 139 c & 344 e & $571 \quad c$ & $364 d$ & $354 \mathrm{~d}$ \\
\hline IAC 1467-296 & 164 c & 267 & $396 \mathrm{~d}$ & 308 & 284 \\
\hline IAC 1467-301 & 198 b & 511 с & 659 c & 455 & 455 c \\
\hline IAC NE-3 & $115 \mathrm{c}$ & 320 e & $321 d$ & 331 & 272 d \\
\hline IAC KM-6 & 192 b & 545 c & 859 b & $728 \quad$ c & 581 b \\
\hline ‘Mizuho’' & $235 \mathrm{~b}$ & 529 c & 737 b & $560 \quad \mathrm{c}$ & $515 \mathrm{~b}$ \\
\hline CV (\%) & 25,32 & 17,81 & 23,12 & 18,21 & 13,07 \\
\hline
\end{tabular}

* Médias não seguidas pela mesma letra na coluna diferem entre si pelo teste Scott-Knott, a 5\% de probabilidade de erro. 
Tabela 2 - Massa fresca média de frutos de novas seleções de nespereira. Centro APTA Frutas/IAC, Jundiaí, SP, 2009.

\begin{tabular}{|c|c|c|c|c|c|}
\hline \multirow{3}{*}{$\begin{array}{l}\text { Seleções } \\
\text { IAC 263-49 }\end{array}$} & \multicolumn{5}{|c|}{-Massa fresca média dos frutos $\left(\mathrm{g} \mathrm{fruto}^{-1}\right)$ - } \\
\hline & 2005 & 2006 & 2007 & 2008 & Média do quadriênio \\
\hline & $25,41 \quad b$ & 25,81 b & 24,53 b & 25,62 b & $25,34 \quad$ c \\
\hline IAC 363-27 & 34,61 a & 27,44 b & 26,12 b & 30,84 а & 29,76 b \\
\hline IAC 264-54 & $23,98 \mathrm{~b}$ & $20,90 \quad c$ & $19,15 \mathrm{~d}$ & $25,78 \mathrm{~b}$ & $22,45 \quad \mathrm{~d}$ \\
\hline IAC 464-9 & $24,71 \mathrm{~b}$ & 23,43 & $19,23 \quad d$ & 24,22 b & 22,90 \\
\hline IAC $165-9$ & $25,11 \mathrm{~b}$ & $21,93 \mathrm{c}$ & 21,04 c & $27,60 \mathrm{~b}$ & 23,92 c \\
\hline IAC 265-66 & 34,01 а & 38,11 a & $26,00 \mathrm{~b}$ & 33,91 a & 33,00 a \\
\hline IAC 966-23 & 36,53 а & 24,99 b & $23,77 \quad$ c & 32,41 a & 29,42 b \\
\hline IAC 167-1 & $27,16 \mathrm{~b}$ & 24,33 b & $22,41 \quad$ c & $28,04 \quad b$ & $25,49 \quad$ c \\
\hline IAC 677-13 & $22,40 \mathrm{~b}$ & $20,91 \quad c$ & $19,99 \mathrm{~d}$ & $23,62 \mathrm{~b}$ & $21,73 \quad d$ \\
\hline IAC 967-5 & $24,57 \mathrm{~b}$ & $24,67 \quad b$ & 24,06 b & $27,68 \quad b$ & 25,24 c \\
\hline IAC 1367-43 & $26,37 \mathrm{~b}$ & $25,12 \mathrm{~b}$ & $22,24 \quad$ c & 24,38 b & 24,53 \\
\hline IAC 1367-46 & $28,35 \mathrm{~b}$ & 32,05 a & $25,60 \mathrm{~b}$ & 28,60 a & 28,65 b \\
\hline IAC $1467-12$ & 24,09 b & 19,77 c & $22,14 \quad$ C & $22,45 \quad b$ & $22,12 \mathrm{~d}$ \\
\hline IAC $1467-25$ & $24,00 \mathrm{~b}$ & $23,11 \quad c$ & $19,98 \quad d$ & 26,02 b & 23,27 \\
\hline IAC $1467-170$ & $30,63 \mathrm{~b}$ & 19,91 c & $22,11 \quad$ c & 26,73 b & 24,85 \\
\hline IAC 1467-177 & $23,46 \mathrm{~b}$ & $15,67 \quad \mathrm{c}$ & $21,00 \quad c$ & $25,81 \mathrm{~b}$ & 21,48 \\
\hline IAC $1467-296$ & $23,53 \mathrm{~b}$ & 25,02 b & 22,53 c & 29,49 a & 25,14 c \\
\hline IAC $1467-301$ & $23,66 \mathrm{~b}$ & 18,12 c & $20,64 d$ & $24,87 \quad b$ & $21,82 \quad \mathrm{~d}$ \\
\hline IAC NE-3 & $30,20 \mathrm{~b}$ & 25,83 b & 30,21 a & 30,14 a & 29,10 b \\
\hline IAC KM-6 & $25,71 \mathrm{~b}$ & $19,06 \quad \mathrm{c}$ & $18,08 \mathrm{~d}$ & 21,51 b & $21,09 \mathrm{~d}$ \\
\hline ‘Mizuho’' & $31,05 \mathrm{~b}$ & 23,77 & $26,11 \quad b$ & 28,45 b & 26,90 \\
\hline CV (\%) & 17,82 & 13,75 & 9,25 & 10,55 & 7,09 \\
\hline
\end{tabular}

* Médias não seguidas pela mesma letra na coluna diferem entre si pelo teste Scott-Knott, a 5\% de probabilidade de erro.

diferindo estatisticamente da nespereira 'Mizuho' (Tabela 3). No segundo ano de avaliação, as três primeiras seleções citadas permaneceram com a maior produção média, já diferindo da 'Mizuho' em aproximadamente $6 \mathrm{~kg}$. Essa diferença aumentou na avaliação realizada em 2008, em que as seleções IAC 165-9, IAC 265-66 e IAC 677-13 registraram as maiores produções, $10 \mathrm{~kg}$ a mais que a nespereira 'Mizuho'. No entanto, levando-se em consideração a média do quadriênio, a seleção IAC 165-9 foi a que se destacou entre as demais. Já as outras seleções não apresentaram desempenho produtivo superior à 'Mizuho'.

As seleções IAC 165-9, IAC 265-66 e IAC 677-13 apresentaram produção superior no quarto ano de avaliação e na média do quadriênio em relação às nespereiras 'Néctar de Cristal', 'Mizauto' e 'Mizumo', que registraram média de produção ao redor de $15 \mathrm{~kg}$ (PIO et al., 2007a). Em pesquisa similar, realizada em Monte Alegre do Sul, SP, a ‘Mizuho’ mostrou produção bem inferior no primeiro quadriênio. Em espaçamento 6x6m (278 plantas ha $\left.{ }^{-1}\right)$, essa cultivar produziu apenas 8,2kg planta ${ }^{-1}$ (OJIMA et al., 1999).
Como foi estimada a produtividade a partir da população de 357 plantas por ha (espaçamento $7 \mathrm{x} 4 \mathrm{~m}$ ), os resultados estatísticos correlacionam-se com a produção por planta (Tabela 4). A seleção IAC 165-9 apresentou 2,84ton a mais que a nespereira 'Mizuho', sendo levada em consideração a média do quadriênio. No entanto, deve-se fazer uma ressalva em relação à quarta avaliação, em que as seleções IAC 165-9, IAC 265-66 e IAC 677-13 chegaram ao redor de 10ton ha-1, praticamente o dobro do valor obtido pela 'Mizuho'. Esses resultados já no quarto ano produtivo são excepcionais, uma vez que a literatura cita que as nespereiras podem chegar a produzir 10 ton $\mathrm{ha}^{-1}$ no oitavo ano de produção, desde que as plantas estejam corretamente manejadas (OJIMA et al., 1999).

\section{CONCLUSÕES}

As seleções IAC 165-9, IAC 265-66 e IAC 677-13 apresentaram maior desempenho produtivo, sendo excelentes opções para a diversificação do cultivo de nêspera no Brasil. 
Tabela 3 - Produção média por planta de novas seleções de nespereira. Centro APTA Frutas/IAC, Jundiaí, SP, 2009.

\begin{tabular}{|c|c|c|c|c|c|}
\hline \multirow{3}{*}{$\begin{array}{l}\text { Seleções } \\
\text { IAC 263-49 }\end{array}$} & \multicolumn{5}{|c|}{ 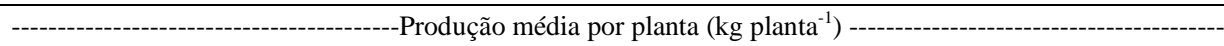 } \\
\hline & 2005 & 2006 & 2007 & 2008 & Média do quadriênio \\
\hline & 2,96 & 5,41 & $13,55 \mathrm{c}$ & 13,77 & 8,92 \\
\hline IAC 363-27 & $2,83 \mathrm{C}$ & 9,70 & $17,75 \mathrm{~b}$ & $19,92 \mathrm{~b}$ & 12,56 \\
\hline IAC 264-54 & 5,26 b & 9,75 & $18,46 \quad b$ & $15,92 \quad \mathrm{c}$ & 12,35 \\
\hline IAC 464-9 & 9,47 a & 18,03 a & $16,20 \quad \mathrm{c}$ & $20,79 \quad b$ & 16,13 \\
\hline IAC $165-9$ & 10,74 a & 18,82 a & 26,32 а & 31,33 a & 21,80 a \\
\hline IAC 265-66 & 7,65 a & 19,93 а & 21,11 a & 28,99 а & 20,16 b \\
\hline IAC 966-23 & 5,04 b & $12,72 b$ & $17,73 \mathrm{~b}$ & $21,23 b$ & $14,18 \quad \mathrm{c}$ \\
\hline IAC 167-1 & $3,63 \quad$ c & 8,49 & $10,31 \quad d$ & $15,61 \quad \mathrm{c}$ & 9,51 \\
\hline IAC 677-13 & 7,85 a & $14,39 \quad b$ & 20,81 a & 26,59 a & $17,41 \quad$ c \\
\hline IAC 967-5 & $4,11 \quad \mathrm{c}$ & 8,74 & $13,16 \quad c$ & $15,22 \quad \mathrm{c}$ & 10,31 \\
\hline IAC 1367-43 & 5,49 b & 10,43 & $12,26 \quad d$ & 14,22 & 10,61 \\
\hline IAC 1367-46 & $6,08 \mathrm{~b}$ & $12,17 \quad b$ & 14,82 с & 16,85 & 12,48 \\
\hline IAC 1467-12 & $4,11 \quad \mathrm{c}$ & 9,19 & $18,01 \quad b$ & 15,72 & 11,76 \\
\hline IAC 1467-25 & 5,32 b & $12,31 \quad b$ & 14,86 с & 14,86 & 11,84 \\
\hline IAC $1467-170$ & 8,30 a & $13,67 \quad b$ & 21,06 a & 15,34 & 14,59 \\
\hline IAC 1467-177 & $3,33 \quad c$ & 5,39 & $12,01 \quad d$ & 9,28 & 7,51 \\
\hline IAC 1467-296 & $4,43 \quad \mathrm{c}$ & 6,68 & 8,93 & 11,18 & 7,80 \\
\hline IAC 1467-301 & $4,62 \mathrm{~b}$ & 8,01 & $13,47 \quad \mathrm{c}$ & 10,98 & 9,27 \\
\hline IAC NE-3 & $3,47 \quad \mathrm{c}$ & 8,45 & $9,45 \quad d$ & 9,89 & 7,81 \\
\hline IAC KM-6 & $4,79 \mathrm{~b}$ & 10,32 & $15,50 \quad \mathrm{c}$ & 15,65 & 11,57 \\
\hline 'Mizuho' & 7,06 а & $12,57 \mathrm{~b}$ & $19,24 \quad b$ & 15,93 & 13,85 \\
\hline CV (\%) & 29,26 & 20,26 & 19,53 & 20,41 & 13,37 \\
\hline
\end{tabular}

* Médias não seguidas pela mesma letra na coluna diferem entre si pelo teste Scott-Knott, a 5\% de probabilidade de erro.

Tabela 4 - Produtividade média por planta de novas seleções de nespereira. Centro APTA Frutas/IAC, Jundiaí, SP, 2009.

\begin{tabular}{|c|c|c|c|c|c|c|}
\hline \multirow{3}{*}{$\begin{array}{l}\text { Seleções } \\
\text { IAC 263-49 }\end{array}$} & \multicolumn{6}{|c|}{--------------------------------------Produtividade média por planta (ton ha'1) -------------------------------------- } \\
\hline & 2005 & 2006 & 2007 & \multicolumn{2}{|c|}{2008} & Média do quadriênio \\
\hline & $1,06 \quad \mathrm{c}$ & $1,93 \mathrm{~d}$ & $4,84 \quad \mathrm{c}$ & 4,92 & $\mathrm{C}$ & 3,19 \\
\hline IAC 363-27 & $1,01 \quad \mathrm{c}$ & $3,46 \quad c$ & $6,34 \mathrm{~b}$ & 7,11 & $\mathrm{~b}$ & 4,48 \\
\hline IAC 264-54 & $1,88 \quad b$ & $3,48 \quad c$ & $6,59 \mathrm{~b}$ & 5,68 & c & 4,41 \\
\hline IAC 464-9 & 3,38 a & 6,44 a & $5,78 \quad$ c & 7,42 & $\mathrm{~b}$ & $5,76 \quad$ c \\
\hline IAC 165-9 & 3,83 a & 6,72 а & 9,40 a & 11,18 a & & 7,78 а \\
\hline IAC 265-66 & 2,73 a & 7,11 a & 7,53 а & $10,34 \mathrm{a}$ & & $7,19 \mathrm{~b}$ \\
\hline IAC 966-23 & $1,79 \mathrm{~b}$ & $4,54 \mathrm{~b}$ & $6,33 \mathrm{~b}$ & 7,58 & $\mathrm{~b}$ & 5,06 c \\
\hline IAC 167-1 & $1,30 \quad \mathrm{c}$ & 3,03 c & $3,68 \mathrm{~d}$ & 5,57 & c & 3,40 \\
\hline IAC 677-13 & 2,80 a & $5,14 \mathrm{~b}$ & 7,43 а & 9,49 a & & 6,22 \\
\hline IAC $967-5$ & $1,47 \quad \mathrm{c}$ & $3,12 \quad \mathrm{c}$ & $4,70 \mathrm{c}$ & 5,43 & c & 3,68 \\
\hline IAC $1367-43$ & $1,96 \mathrm{~b}$ & $3,72 \quad \mathrm{c}$ & 4,38 & 5,08 & c & 3,78 \\
\hline IAC 1367-46 & $2,17 \quad b$ & $4,34 \mathrm{~b}$ & $5,29 \quad \mathrm{c}$ & 6,02 & c & 4,46 \\
\hline IAC 1467-12 & $1,47 \quad \mathrm{c}$ & $3,28 \quad c$ & $6,43 \quad b$ & 5,61 & $\mathrm{C}$ & 4,20 \\
\hline IAC 1467-25 & $1,90 \quad b$ & 4,39 b & $5,30 \quad \mathrm{c}$ & 5,30 & C & 4,22 \\
\hline IAC 1467-170 & 2,96 a & $4,88 \mathrm{~b}$ & 7,52 a & 5,48 & c & 5,21 \\
\hline IAC 1467-177 & 1,19 c & $1,92 \quad d$ & $4,29 \quad \mathrm{~d}$ & 3,31 & $\mathrm{~d}$ & 2,68 \\
\hline IAC 1467-296 & $1,58 \quad \mathrm{c}$ & 2,38 & 3,19 & 3,99 & $\mathrm{~d}$ & 2,79 \\
\hline IAC 1467-301 & $1,65 \mathrm{~b}$ & 2,86 & $4,81 \quad c$ & 3,92 & d & 3,31 \\
\hline IAC NE-3 & 1,24 c & 3,02 & $3,37 \quad d$ & 3,53 & $\mathrm{~d}$ & 2,79 \\
\hline IAC KM-6 & $1,71 \quad b$ & $3,68 \quad$ c & 5,53 с & 5,59 & c & 4,13 \\
\hline ‘Mizuho’’ & 2,52 a & $4,48 \quad b$ & $6,86 \mathrm{~b}$ & 5,68 & c & 4,94 \\
\hline CV (\%) & 29,26 & 20,26 & 19,53 & 20,41 & & 13,37 \\
\hline
\end{tabular}

* Médias não seguidas pela mesma letra na coluna diferem entre si pelo teste Scott-Knott, a 5\% de probabilidade de erro. 


\section{REFERÊNCIAS}

AGUSTÍ, M. et al. Effect of the synthetic auxin 2,4-DP on fruit development of loquat. Plant Growth Regulation, v.41, p.129-132, 2003.

BARBOSA, W. et al. Distribuição geográfica e diversidade varietal de frutíferas e nozes de clima temperado no Estado de São Paulo. Revista Brasileira de Fruticultura, v.25, n.2, p.341-344, 2003. Disponível em: <http://www.scielo.br/ s c i elo.ph p s c ri p t = s ci_art tex t \& pid = S 0100 29452003000200042\&lng=pt\&nrm=iso $>$. Acesso em: 08 jan. 2010. doi: 10.1590/S0100-29452003000200042.

EMBRAPA. Centro Nacional de Pesquisa de Solos. Sistema Brasileiro de Classificação de Solos. 2.ed. Rio de Janeiro: EMBRAPA/SOLOS, 2006. 306p.

FARIA, A.F. et al. Cultivar influence on carotenoid composition of loquats from Brazil. Journal of Food Composition and Analysis, v.22, p.196-203, 2009. Disponível em: <http:// w w w. s c i e n c e d i r e c t. c o m / science?_ob=ArticleURL\&_udi=B6WJH-4VBDKCF-

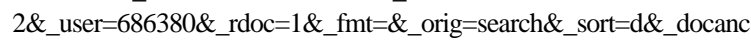
hor $=\&$ \&view $=$ c\&_searchStrId $=1159287229 \&$ _rerunOrigin $=$ google\&_acct $=C$ $000037521 \&$ \&_version $=1 \&$ \&_urlversion $=0 \&$ \&_use rid $=686380 \&$ md5 $=$ ae75b18aab32a225fd75e26569b21b54 $>$. Acesso em: 08 jan. 2010. doi: 10.1016/j.jfca.2008.10.014.

FERREIRA, D.F. Análise estatística por meio do SISVAR (Sistema para Análise de Variância) para Windows versão 4.0. In: REUNIÃO ANUAL DA REGIÃO BRASILEIRA DA SOCIEDADE INTERNACIONAL DE BIOMETRIA, 45., 2000, São Carlos. Anais... São Carlos: UFSCar, 2000. p.255258.

FERRERES, F. et al. Improved loquat (Eriobotrya japonica Lindl.) cultivars: variation of phenolics and antioxidative potential. Food Chemistry, v.114, p.1019-1027, 2009. Disponível em: <http:// www.sciencedirect.com/science?_ob=ArticleURL\&_udi=B6T6R4TVHSV5-C\&_user=686380\&_rdoc $=1 \&$ fmt $=\&$ \&orig=search $\&$ so $\mathrm{rt}=\mathrm{d} \&$ \&docanchor $=\&$ view $=$ c \&_searchStrId=115929 2465\&_rerunOrigin=google\&_acct $=$ C000037521\&_versi o n $=1 \&$ _ url Versio $n=0 \&$ _ us e ri d $=686380$ \&md5=389fa6e7289961f4ea7d3041ab88c4dd $>$. Acesso em: 08 jan. 2010. doi: 10.1016/j.foodchem.2008.10.065.

FEMENIA, A. et al. Characterization of the cell walls of loquat (Eriobotrya japonica) fruit tissues. Carbohydrate Polymers, v.35, n.1/2, p. 169-177, 1998. Disponível em: <http:// www.sciencedirect.com/science?_ob=ArticleURL\&_udi=B6TFD3VY60VK-5\&_user $=686380 \& \_$rdoc $=1 \&$ fmt $=$ \&_orig $=$ search \& sort $=$ d\&_docanchor $=\&$ view $=$ c \&_searchStrId $=1159290$ $679 \&$ \&erunOrigin=google $\&$ _acct $=$ C $000037521 \&$ \&vers i o $n=1 \&$ _ url Version $=0 \&$ _ us erid $=686380 \& \mathrm{~m}$ d5=a7a6a062aa43a9bd78e457bd5f3e6058>. Acesso em: 08 jan. 2010. doi: 10.1016/S0144-8617(97)00240-3.

GRASSI, A.M. et al. Avaliação na intensidade de raleio na qualidade de frutos de nespereira. Bragantia, v.69, n.1, p. 215-220, 2010. Disponível em: <http://www.scielo.br/ scielo.php ? script=sci_art text \& pid=S 0006 $87052010000100027 \& \operatorname{lng}=p t \& n r m=i s o \& t \operatorname{lng}=p t>$. Acesso em: 20 maio, 2010. doi: 10.1590/S0006-87052010000100027.

KOBA, K. et al. Effect of loquat (Eriobotrya japonica) extracts on LDL oxidation. Food Chemistry, v.104, p.308316, 2007. Disponível em: <http://www.sciencedirect.com/ science?_ob=ArticleURL\&_udi=B6T6R-4NC5V9H18 _user $=686380 \&$ \&doc $=1 \&$ \& fmt $=$ \&_orig $=$ search\&_sort $=\mathrm{d} \&$ _ d oc anchor $=\&$ view $=$ c \&_searchStrId $=1159290322$ $\&_{\text {_rerunOrigin }}$ google \&_acct $=$ C 0000375 $21 \&$ _version $=1 \&$ _ ur l Version $=0 \&$ _useri d $=$ 686380 \&md5 $=c 446062$ aacf3afa50ade21503b54bad6 $>$. Acesso em: 08 jan. 2010. doi: 10.1016/j.foodchem.2006.11.043.

MELO, A.A.M.; LIMA, L.C.O. Influência de três diferentes embalagens de PVC na vida pós-colheita de nêspera. Ciência e Agrotecnologia, v.27, n.6, p.1330-1339, 2003.

OJIMA, M. et al. Cultura da nespereira. Campinas: Instituto Agronômico, 1999. 36p. (Boletim técnico, 185).

PEROSA, J.M. et al. Cadeia produtiva da nêspera na região do Alto do Tietê: indicadores econômicos da produção e mercado atacadista. Revista Brasileira de Fruticultura, v.28, n.2, p.214-217, 2006. Disponível em: <http://www.scielo.br/ scielo.php? script=s ci_art tex t \& pi d = S 0100 29452006000200013\&lng=pt\&nrm=iso>. Acesso em: 08 jan. 2010. doi: 10.1590/S0100-29452006000200013.

PIO, R. et al. Produção de cultivares de nespereira na região Leste paulista. Pesquisa Agropecuária Brasileira, v.42, n.7, p.1053-1056, 2007a. Disponível em: <http://www.scielo.br/ scielo.php? script=s ci_art text\&pid=S 0100 204X2007000700020\&lng=pt\&nrm=iso $>$. Acesso em: 08 jan. 2010. doi: 10.1590/S0100-204X2007000700020.

PIO, R. et al. Desempenho produtivo de nespereiras enxertadas em marmeleiro 'Portugal'. Pesquisa Agropecuária Brasileira, v.42, p.1715-1719, 2007b. Disponível em: <http:/ /www.scielo.br/scielo.php?script=sci_arttext\&pid=S0100204X2007001200007\&lng=pt\&nrm=iso $>$. Acesso em: 08 jan. 2010. doi: 10.1590/S0100-204X2007001200007.

PIO, R. et al. Aspectos técnicos do cultivo de nêsperas. Piracicaba: DIBD/ESALQ/USP, 2008. 30p. (Série Produtor Rural, 39). 